\title{
Cyclic intensive light exposure induces retinal lesions similar to age-related macular degeneration in APPswe/PS1 bigenic mice
}

\author{
Zhizhang Dong ${ }^{1}$, Juan $\mathrm{Li}^{1}$, Yunxia Leng ${ }^{1}$, Xuerong Sun ${ }^{1}$, Huiling Hu${ }^{1}$, Yuan $\mathrm{He}^{1}$, Zhiqun $\operatorname{Tan}^{2,3^{*}}$ and Jian Ge ${ }^{1 *}$
}

\begin{abstract}
Background: Intensive light exposure and beta-amyloid (A $\beta$ ) aggregates have been known as a risk factor for macular degeneration and an important component in the pathologic drusen structure involved in this disorder, respectively. However, it is unknown whether $A \beta$ deposition mediates or exacerbates light exposure-induced pathogenesis of macular degeneration. Several studies including the one from us already showed accumulation of $A \beta$ deposits in the retina in Alzheimer's transgenic mice. Using histopathological analysis combined with electroretinographic functional assessment, we investigated the effects of cyclic intensive light exposure (CILE) on the architecture of retina and related function in the APPswe/PS1 bigenic mouse.

Results: Histopathological analysis has found significant loss of outer nuclear layer/photoreceptor outer segment and outer plexiform layer along with abnormal hypo- and hyper-pigmentation in the retinal pigment epithelium (RPE), remarkable choroidal neovascularization (CNV), and exaggerated neuroinflammatory responses in the outer retina of APPswe/PS1 bigenic mice following cyclic intensive light exposure (CILE), whereas controls remained little change contrasted with age-matched non-transgenic littermates. CILE-induced degenerative changes in RPE are further confirmed by transmission electron microcopy and manifest as formation of basal laminar deposits, irregular thickening of Bruch's membrane (BrM), deposition of outer collagenous layer (OCL) in the subretinal space, and vacuolation in the RPE. Immunofluorescence microscopy reveals drusenoid A $\beta$ deposits in RPE as well as neovessels attached which are associated with disruption of RPE integrity and provoked neuroinflammatory response as indicated by markedly increased retinal infiltration of microglia. Moreover, both immunohistochemistry and Western blots detect an induction of vascular endothelial growth factor (VEGF) in RPE, which corroborates increased CNV in the outer retina in the bigenic mice challenged by CILE.

Conclusions: Our findings demonstrate that degenerative changes in the outer retina in the APPswe/PS1 bigenic mouse induced by CILE are consistent with these in AMD. These results suggest that an Alzheimer's transgenic animal model with accumulation of $A \beta$ deposits might be an alternative animal model for AMD, if combined with other confounding factors such as intensive light exposure for AMD.
\end{abstract}

\section{Background}

Age-related macular degeneration (AMD) is a degenerative disease in the eye, which causes irreversible blindness in elderly and is one of the major causes of blindness in developed countries [1]. Drusen and

\footnotetext{
* Correspondence: tanz@uci.edu; gejian@mail.sysu.edu.cn

'State Key Laboratory of Ophthalmology, Zhongshan Ophthalmic Center,

Sun Yat-sen University, Guangzhou, China

${ }^{2}$ Department of Neurology, University of California Irvine School of Medicine, Irvine, CA 92697, USA

Full list of author information is available at the end of the article
}

choroidal neovascularization (CNV) are the two pathological hallmarks of AMD, of which drusen accumulates in the subretinal pigment epithelium (RPE) space and $\mathrm{CNV}$ is characterized by new angiogensis from choroidal blood vessels which break through Bruch's membrane $(\mathrm{BrM})$ and RPE layer and is often associated with subretinal hemorrhage [2]. Recent studies suggest that betaamyloid $(A \beta)$ peptide, a major molecular signature in the brain of Alzheimer's disease, might play an important role in the pathogenesis of AMD [3]. A $\beta$ aggregates have been identified as one of the major components in
C Biomed Central

() 2012 Dong et al; licensee BioMed Central Ltd. This is an Open Access article distributed under the terms of the Creative Commons Attribution License (http://creativecommons.org/licenses/by/2.0), which permits unrestricted use, distribution, and reproduction in any medium, provided the original work is properly cited. 
drusen as well as in RPE cells in the retina of AMD [4-7]. Similarly to the brain, several groups of investigators including us also demonstrate perivascular deposition of $A \beta$ in the retina in human $C N V$ as well as different lines of Alzheimer's-related transgenic mice $[8,9]$. Importantly, immunotherapy that targets $A \beta$ significantly attenuated retinal lesions and improved retinal function in an AMD mouse model [10,11]. Moreover, growing evidence has indicated smoking [12], extensive sun light exposure [13], and ageing [14] as important risk factors for AMD. CILE is detrimental to the BrM, RPE, photoreceptor and other retinal structures due to induction of the reactive oxygen species and inflammatory response $[15,16]$. CILE induced drusen formation or stimulated CNV through upregulation of vascular endothelial growth factor (VEGF) as well as induction of oxidative stress in rodent models [17-20].

Nevertheless, the molecular basis of the pathogenesis of $A M D$, particularly about the role of $A \beta$ deposition in the development of RPE lesions and CNV, remains elusive. In this study we examined the effects of constitutional expression of $A \beta$ deposits on retinal lesions induced by CILE in the APPswe/PS1 bigenic mouse model of Alzheimer's disease, and found that CILE significantly increased $A \beta$ deposition linked with AMD-like retinopathies in the transgenic mice. By contrast, there were no significant changes in the retina of either non-transgenic mice received equal light exposure or age-matched transgenic control.

\section{Results \\ Cyclic intensive light exposure induces abnormal pigment deposition in RPE, CNV and degenerative changes in the retina of APPswe/PS1 bigenic mice}

To evaluate the effect of CILE on the retina of mice, the fundus was examined before and after CILE based on fundus photographs. Apparently, increased pigment deposits and shrunken vessels were detected in APPswe/ PS bigenic mice after CILE, particularly in these after 6-month CILE compared with age-matched control or non-Tg mice after the exposure (Additional file 1, Figure 6). Nevertheless, neither yellowish retinal deposits/drusen nor retinal hemorrhage was found in the fundus photos from both bigenic and non-Tg mice. These observations are in agreement with conventional light microscopic analysis following H\&E staining on retinal cross sections (Figure 1). There is no conspicuous difference in the architecture of the retina between a non-Tg (Figure 1A) and an age-matched bigenic control (Figure 1B) mouse. By contrast, a series of remarkable degenerative changes are visible in the retina in all the animals from the groups of bigenic mice following CILE (Figures 1C-J) compared with the control (Figure 1B). Significant loss of the outer nuclear layer $(\mathrm{ONL})$ /photoreceptors is visible following 3-month
CILE (Figure 1C), the entire outer plexiform layer (OPL) and ONL/photoreceptor outer segment (OS) layer are barely remaining after 6-month CILE (Figures 1D, H, and 1I), which is consistent with the thickness loss as confirmed by thickness measurement (Figure $1 \mathrm{~K}$ ). Importantly, abnormal pigment deposition, i.e., hypopigmentation (Figure 1E) or hyperpigmentation which appears in association with RPE proliferation as indicated by the hypertrophic appearance of RPE cells in the retina in the bigenic mice after 6-month CILE (Figure 1F). Moreover, CILE-induced CNV is usually shown here as the disruption of the Bruch's membrane (BrM) due to invasion of new vessels that enclose red blood cells from choroicapillaris (CC) (Figures 1D, G-J). Quantification of newly generated vessels shows $75 \%$ of the mice ( 9 of 12) with about $4.8 \pm 2.2$ evident new vessels per cross retinal section in the group with 6 -month CILE, but no new vessel was detected in the other groups at all (Figure 1L). In addition, no typical drusen structures were found in these mice.

To evaluate the CILE-induced pathological lesions in the retina further, we performed transmission electron microscopy to examine the ultrastructure in the retina. A representative micrograph demonstrates typical normal ultrastructure of the outer retina in the 12-month old non-Tg mice (Figure 2A). There is no obvious difference in the general ultrastructure as well as the RPE-BrM-CC complex between non-Tg and age-matched APPswe/PS1 bigenic controls (Figure 2B). Conversely, intracellular vacuoles along with membranous debris (Figures $2 \mathrm{C}, \mathrm{D}$ ), small basal laminar deposits (Figure 2E), and thickened BrM (Figures 2C, F) are commonly seen in the RPE layer in the group of bigenic mice that received 6-month CILE. Noticeably, shrunken and disrupted architecture of basal infoldings of RPE is also detected in most eyes from these animals as well (Figures 2F, G, and 2J). In some cases, loss of the continuity of BrM elastic layer (Figure $2 \mathrm{G}$ ), outer collagenous layer deposition (Figure $2 \mathrm{H}$ ), and infiltration of microglia (Figures $2 \mathrm{G}$ and $2 \mathrm{I}$ ) around the BrM-CC complex are evident as previously demonstrated $[21,22]$. Importantly, CILE also induces invasion of choroicapillaris into the RPE layer resulting in formation of neovessels, which usually demonstrate as vascular buds as shown in Figures 2I and 2J.

\section{Cyclic intensive light exposure-induced retinal lesions are associated with accumulation of $\beta$-amyloid deposits in the RPE of APPswe/PS1 bigenic mice}

Previous studies have demonstrated a link between accumulation of $\mathrm{A} \beta$ deposits and retinal degenerative changes in age-related macular degeneration [5,6]. Both APP and $\mathrm{A} \beta$ deposits have also been found in the retina of APPswe/PS1 bigenic mouse (Additional file 1, Figure 7), we therefore investigated whether CILE-induced retinal lesions are directly associated with retinal accumulation of 

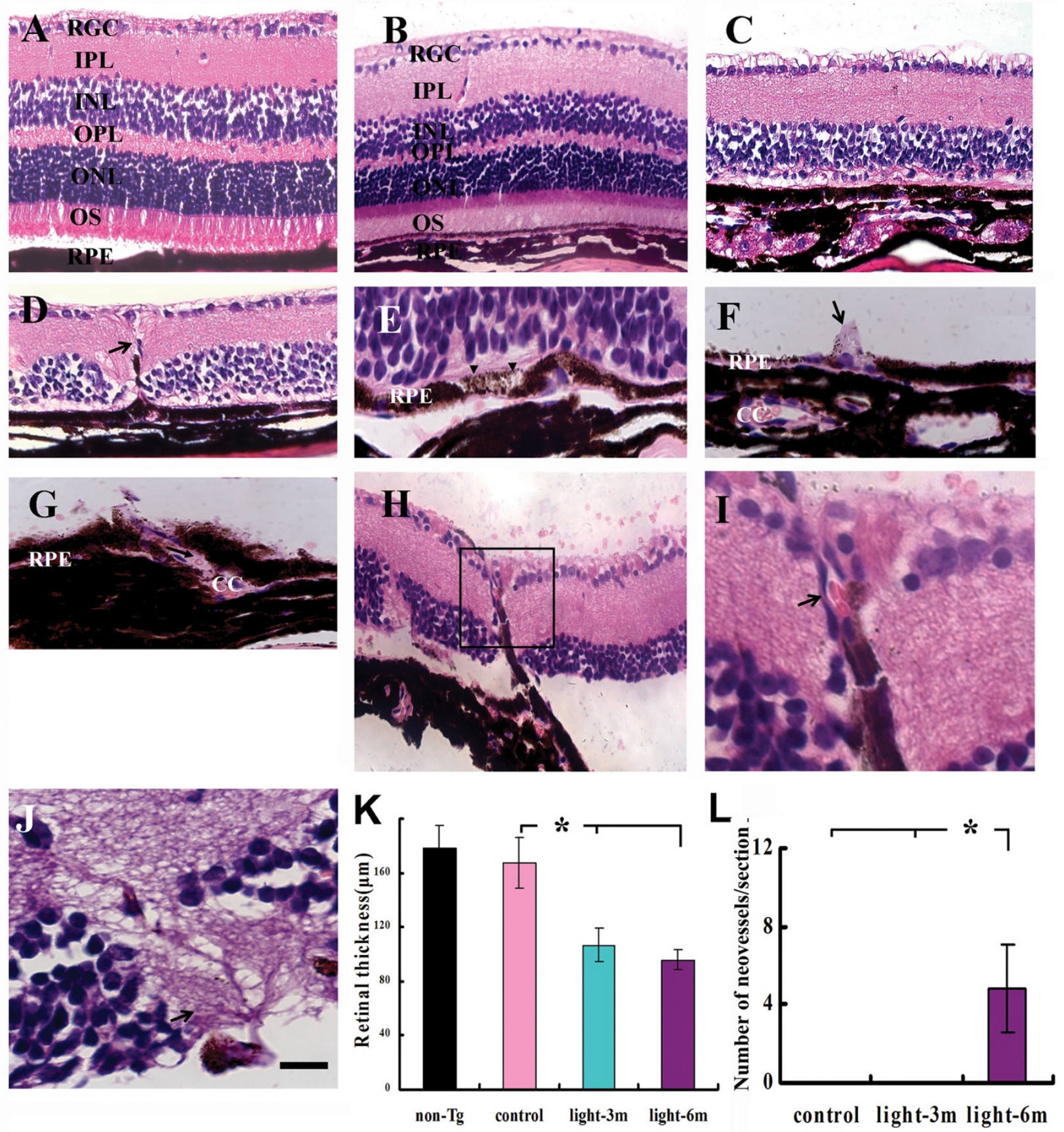

Figure 1 Degenerative changes and neovascularization in the retina of bigenic mice following cyclic intensive light exposure Hematoxylin and eosin ( $H$ \& E) staining was performed on retinal cross sections as described in Methods. (A) A part of a retinal cross section through the optic nerve head of a 12-month old non-Tg control mouse (non-Tg) showing normal architecture and morphology. (B) A part of a retinal cross section from an age-matched APPswe/PS1 bigenic control mouse (control) (demonstrates similar morphology to the non-Tg control). (C) A part of a retinal cross section from a bigenic mouse following 3-month CILE (light-3 $\mathrm{m}$ ) shows remarkable thinning outer nuclear layer/photoreceptor outer segment. (D) A part of a retinal cross section from a bigenic mouse following 6-month CILE (light-6 m) exhibits thinning neuroepithelial layers and newly-developed vessels. (E-G) Cross sections from the bigenic mice after 6-month CILE showing hypopigmentation ( $E$, arrowheads), proliferating RPE ( $F$, arrow), hyperpigmentation and a newly-developed vessel originated from choroicapillaris disrupt the RPE layer with red blood cells inside (G, arrow) in the RPE layer. (H-J) Show different types of newly-developed vessels that disrupt retinal architecture. Boxed area in $(\mathrm{H})$ is shown in $(\mathrm{I})$ at a higher magnification, in which the arrow indicates a red blood cells in the newlydeveloped vessel. (K) Quantification of the retinal thickness for each group of animals. (L) Quantification of neovessels in the outer retina on the cross section for each group of animals. Bars depict mean \pm SEM. *: P $<0.001$ ( $N=6-8$ ). CC: choroicapillaris; RPE, retinal pigment epithelium; OS, outer segment; ONL, outer nuclear layer; OPL, outer plexiform layer; INL, inner nuclear layer; IPL, inner plexiform layer; RGC, retinal ganglion cell layer. Scale bars $=50 \mu \mathrm{m}$ for (A-D), $20 \mu \mathrm{m}$ for $E, F, G, J, 50 \mu \mathrm{m}$ for $\mathrm{H}, 15 \mu \mathrm{m}$ for I. 


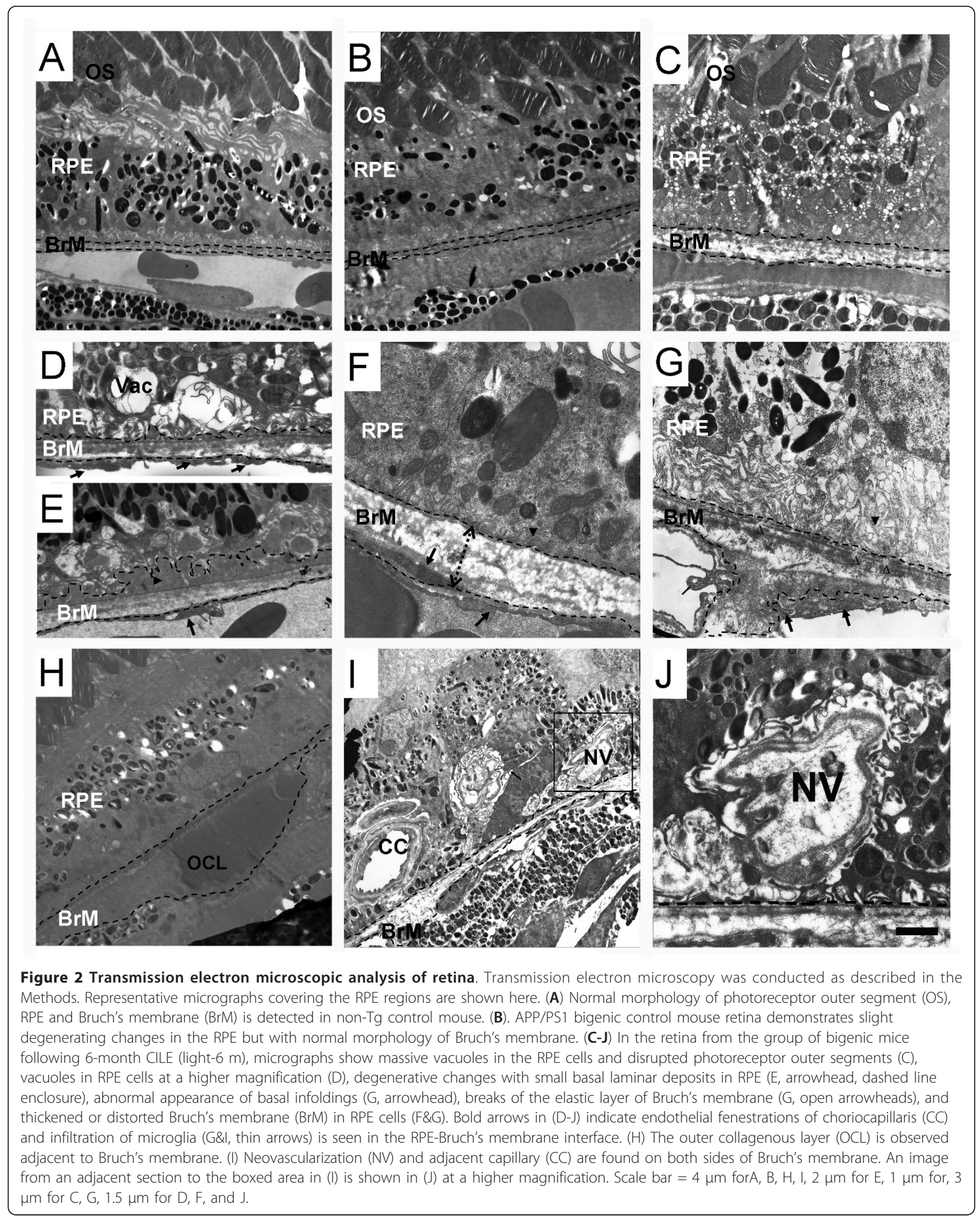


A $\beta$. As shown previously [23], immunohistochemistry following labeling with a specific antibody 22C11 against for three major isoforms of human APP, reveals robust immunoreactivity in the retinal cross sections in the APPswe/ PS1 bigenic mice with 6-month CILE (Additional file 1, Figure $7 \mathrm{C}$ ), whereas only moderate APP staining and a relatively low background is found in the age-matched controls and non-Tg mice (Additional file 1, Figures 7A, B). As speculated, increased APP abundance in the retina is also followed by an increase in accumulation of $A \beta$ deposits with CILE in the RPE as visualized by immunofluorescence microscopy following staining with $6 \mathrm{E} 10$ antibody (Figures 3A-F), which predominantly recognizes $\mathrm{A} \beta$ peptides in the current staining protocol as described [8], though 6E10 can bind to other APP fragments containing $A \beta 1-16$ epitope. Pathological deposition of $A \beta$ in the RPE shares very similar pattern to $A \beta$ plaques in the brain, appearing diffusive (Figure $3 \mathrm{C}$ ), vasculature-associated (data not shown) and/or with a condensed core (Figure 3F) as shown by confocal Z-stack microscopy.

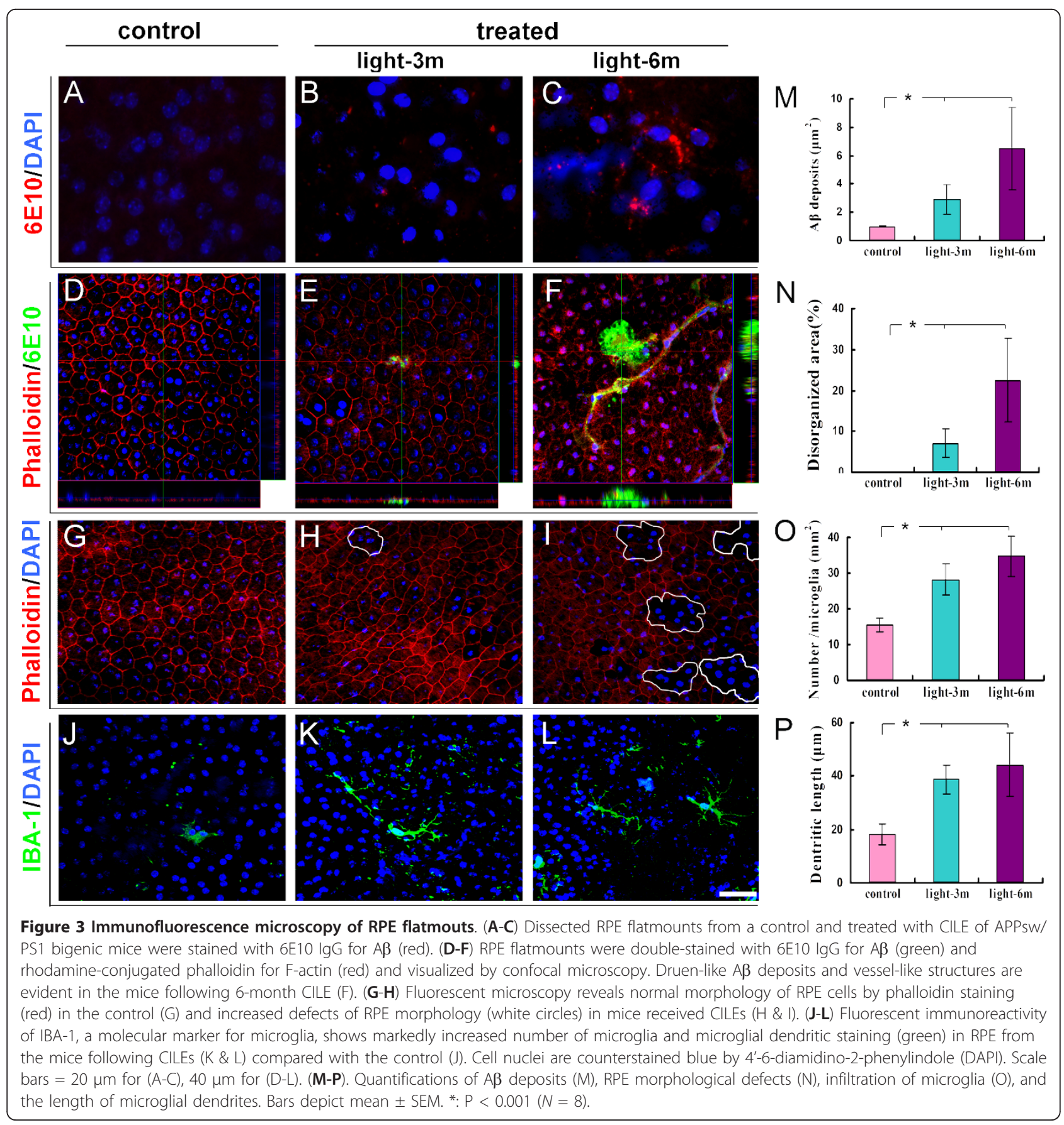


Quantification of $A \beta$ immunoreactivity on the RPE flatmounts demonstrate a significant induction of $A \beta$ deposits by CILE (Figure 3M).

Since CILE as well as accumulation of $A \beta$ either intraor extra-cellular has been linked with oxidative stress and subsequent cell damage [24], we next examined the relationship between CILE and the general architectural integrity of RPE. Accordingly, rhodamine-conjugated phalloidin was used to stain F-actin, a membrane protein that is directly relevant to the cell-cell junctions and is commonly used for assessment of junctions of cells and RPE integrity. Fluorescence microscopy demonstrates well-organized typical hexagonal shape of RPE cells in the control (Figure 3G), but disrupted staining pattern in mice that were treated with CILE (Figures $3 \mathrm{H}$ and 3I). Similar defect in F-actin staining is also obvious in the $\mathrm{A} \beta$-immunoreactive regions (Figure 3F). Quantification of F-actin-unstained area shows a significant increase in loss of RPE integrity with CILE (Figure 3N).

\section{Cyclic intensive light exposure provokes neuroinflammatory response in the RPE layer in APPswe/ PS1 bigenic mice}

Our previous study demonstrated a link of $A \beta$ deposition with exaggerated neuroinflammatory response in the retina of an Alzheimer's mouse [8]. As CILE significantly enhances accumulation of $A \beta$ in the RPE layer, we further examined whether there is a corresponding change in the inflammatory process. Following immune-labeling with an antibody against IBA-1, a molecular marker for microglia, in the RPE flatmounts, fluorescence microscopy demonstrates notably increased immunoreactivity of IBA-1 in the $\mathrm{RPE} /$ choroid complex from the two groups of bigenic mice received CILE compared with these age-matched controls (Figures 3J-L). In addition to more cells that were stained by IBA-1, there was also a change in appearance in which IBA-1 immunoreactive cells exhibited a hypertropic appearance with much more and longer processes in the flatmounts of CILE treated mice (Figures 3J, L). Quantification of IBA-1-positive cells and total length of IBA-1positive dendrites in examined areas indicates a significant increase in the number of microglia and the length of their dendritic processes (Figures $3 \mathrm{O}$ and $3 \mathrm{P}$ ), suggesting an exacerbated inflammatory response with provoked activation of microglia in the RPE in the APPswe/PS bigenic mice after CILE.

\section{Upregulation of VEGF in the RPE-Choroid is related with neovascularization in the retina in APPswe/PS1 bigenic mice following cyclic intensive light exposure}

VEGF has been considered as a major player in angiogenesis and an important mediator in the pathogenesis of "wet" AMD $[25,26]$. To examine whether neovascularization in the outer retina induced by the CILE was on account of abnormal induction of VEGF, immunohistochemistry using a specific antibody against VEGF was performed on retinal cross sections and examined by microscopy following $\mathrm{ABC}$-mediated $\mathrm{AEC}$ staining. When a very low basal level of VEGF signal is detected in the outer retina region from 12-month old non-Tg mice (Figure 4A), a lightly higher level of VEGF immunoreactivity is visible in the APPswe/PS1 bigenic littermates (Figure $4 \mathrm{~B})$. By contrast, robust staining is obvious in the 6-month CILE group (Figure 4C). Quantification of the staining results shows statistical significance of an increase in VEGF immunoreactivity in the 6-month group of CILE relative to the non-Tg as well as bigenic controls. An identical trend of VEGF expression is confirmed by Western blots (Figure 4E).

\section{Excessive light exposure-induced retinal lesions are associated with the functional deficits in APPswe/PS1 bigenic mice}

To investigate the possibility of functional deficits as a result of CILE-induced retinal lesions, all three groups of mice were tested by scotopic ERG recording before euthanasia. Figure 5 illustrates both scotopic rod responses and maximum amplitudes after flash from three tested groups of mice. While no obvious change in $a$ wave can be identified among the three groups of tested mice as the amplitudes stayed at a very low level of background, the rod response shows a conspicuous reduction in the amplitudes of $b$ wave in both groups of mice received 3- or 6-month CILEs in contrast with control mice (Figure 5A), A striking decrease is also detected in the maximum amplitudes flash-triggered $b$ waves in the two groups of treated mice compared with control (Figure 5B). Quantification of both scotopic rod response and the flash-triggered maximum amplitudes of $b$ wave confirms the statistically significant reduction with the time of CILE. But the latency periods of $b$ wave remain relatively stable in both rod and flashinduced maximum response among the three tested groups, as the implicit time stays almost unchanged in all the groups.

\section{Discussion}

Studies have demonstrated abnormal light exposure as one of important pathogenic factors for retinopathies $[15,27,28]$, particularly for inflammatory lesions and RPE degeneration $[29,30]$ in the retina. Here we show that intensive light exposure exacerbates retinal degeneration with significantly exaggerated local neuroinflammatory response along with robust accumulation of $A \beta$ deposits and $\mathrm{CNV}$ in the compartment of outer retina in an Alzheimer's transgenic mouse model. Although neither a typical drusen structure nor lipofuscin buildup has been identified in the model, these pathological changes are directly associated with remarkable functional deficits demonstrated by 


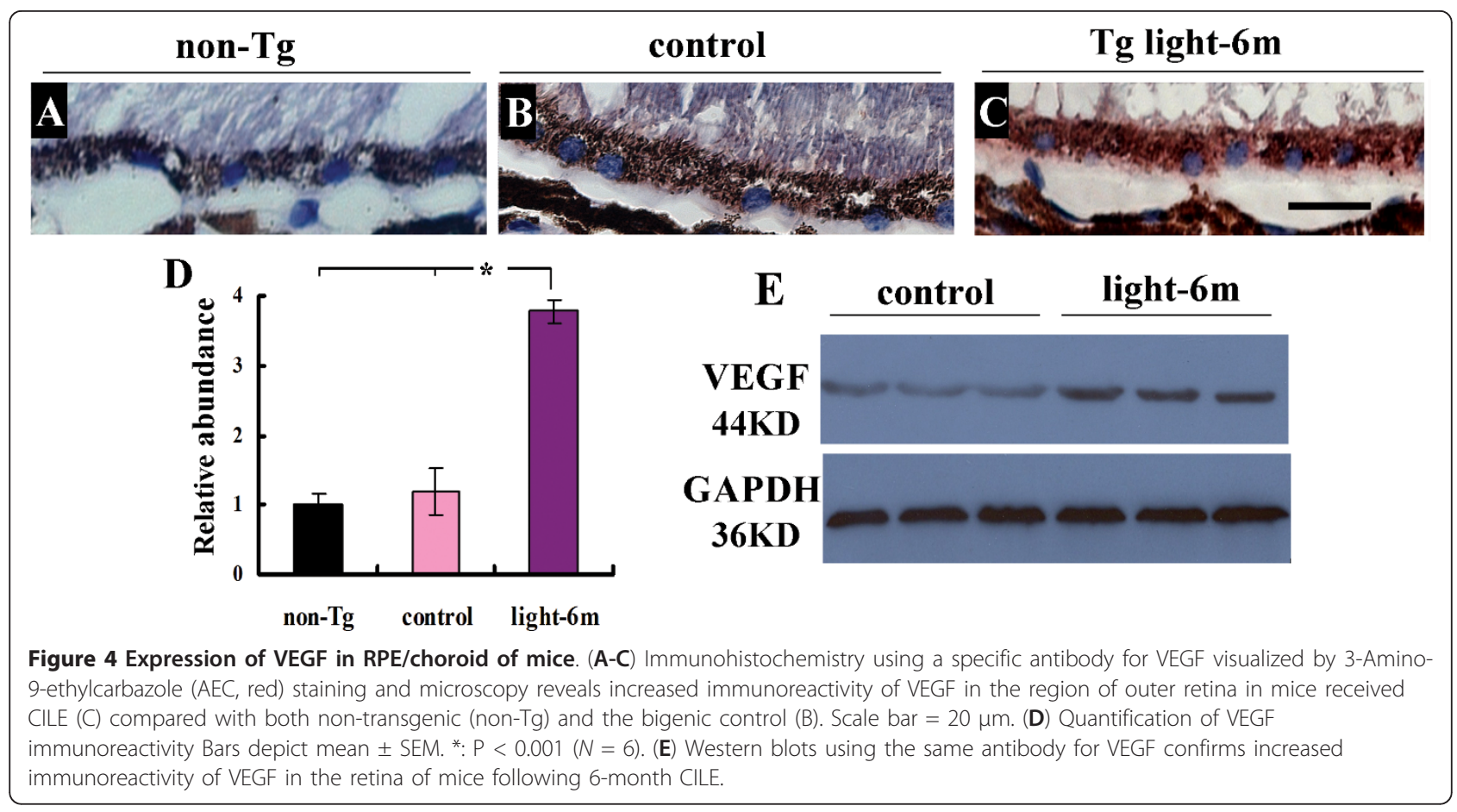

ERG recordings and well mimicked the retinopathies in AMD in human. In addition being known as a very important player in Alzheimer's disease [31,32], A $\beta$ aggregates have been identified as a component of the drusen in AMD $[6,33]$. Reports from several research groups including us has evidenced deposition of $\mathrm{A} \beta$ aggregates in the retina in Alzheimer's transgenic mice [8,23,34], however, the data presented here are the first demonstration of not only an enhancement of $A \beta$ aggregates by phototoxicity in the retina but also a strong link between $A \beta$ deposition and $\mathrm{CNV}$ along with disrupted RPE integrity in the outer retina. Although a variety of animal models have been developed for the study of AMD, they still have their limitation to precisely recapitulate both pathological features and functional deficits of this common visual disorder. Increasing evidence has indicated an important role of $A \beta$ in angiogenesis through induction of VEGF in Alzheimer's brain $[35,36]$. Interestingly, $A \beta$ deposition was also associated with both drusenoid deposits and CNV found in the outer retina in a human apolipoprotein E4 (apoE4) knockin mouse line fed with high fat cholesterol-rich diet [9] as well as other Alzheimer's transgenic mouse lines that result in human $A \beta$ accumulation in both brain and retina (Tan's group, unpublished observations). Furthermore, anti-A $\beta$ immunotherapy protected against loss of $\mathrm{RPE}$ and functional performance in the apoE4 knockin mouse challenged by high fat cholesterol-rich diet [11]. Taken together, our findings further corroborate the notion about an important role of $A \beta$ in the pathogenesis of AMD, particularly in relevance for signal transduction of CNV. In this regard, our study also suggests that an Alzheimer's transgenic mouse model with overexpression of human $A \beta$ deposits in the retina might be a valuable animal model for AMD, when combined with other risk factors such as intensive light exposures for AMD.

Our study also revealed significant exacerbation of neuroinflammatory response in the outer retina of APPswe/ PS1 bigenic mice resulting from CILE, which is in agreement with previous findings about activation of microglia in association with photoreceptor degeneration induced by intensive light exposure [37,38]. Retinal infiltration of microglia could be the crucial player to mediate the loss of photoreceptors and adjacent retinal neurons [38], since anti-inflammatory strategies can efficiently against this type of phototoxicity to the retina [39]. Importantly, $\mathrm{A} \beta$ has shown directly activating microglia both in vivo and in vitro [40-42]. It is therefore possible that intensive light exposure-induced retinopathies might occur through the $A \beta$ approach. Indeed, we also detected a moderate level of APP induction in the outer retina in non-Tg mice following CILE (Figure 7, Additional file 1), though the level of $A \beta$ is still relatively low (Figure $4 A$ ). In comparison with non-Tg mice, in fact, 6-month CILE has significantly removed photoreceptors along with adjacent outer plexiform layers (Figures 1E-J) thereby physically disabled the retinal response to light stimuli as demonstrated in ERG recording (Figure 5B). Nevertheless, retinal infiltration of microglia may be mediated through microglia-Muller cell interaction in the retina [43]. Further studies are therefore warranted to further 


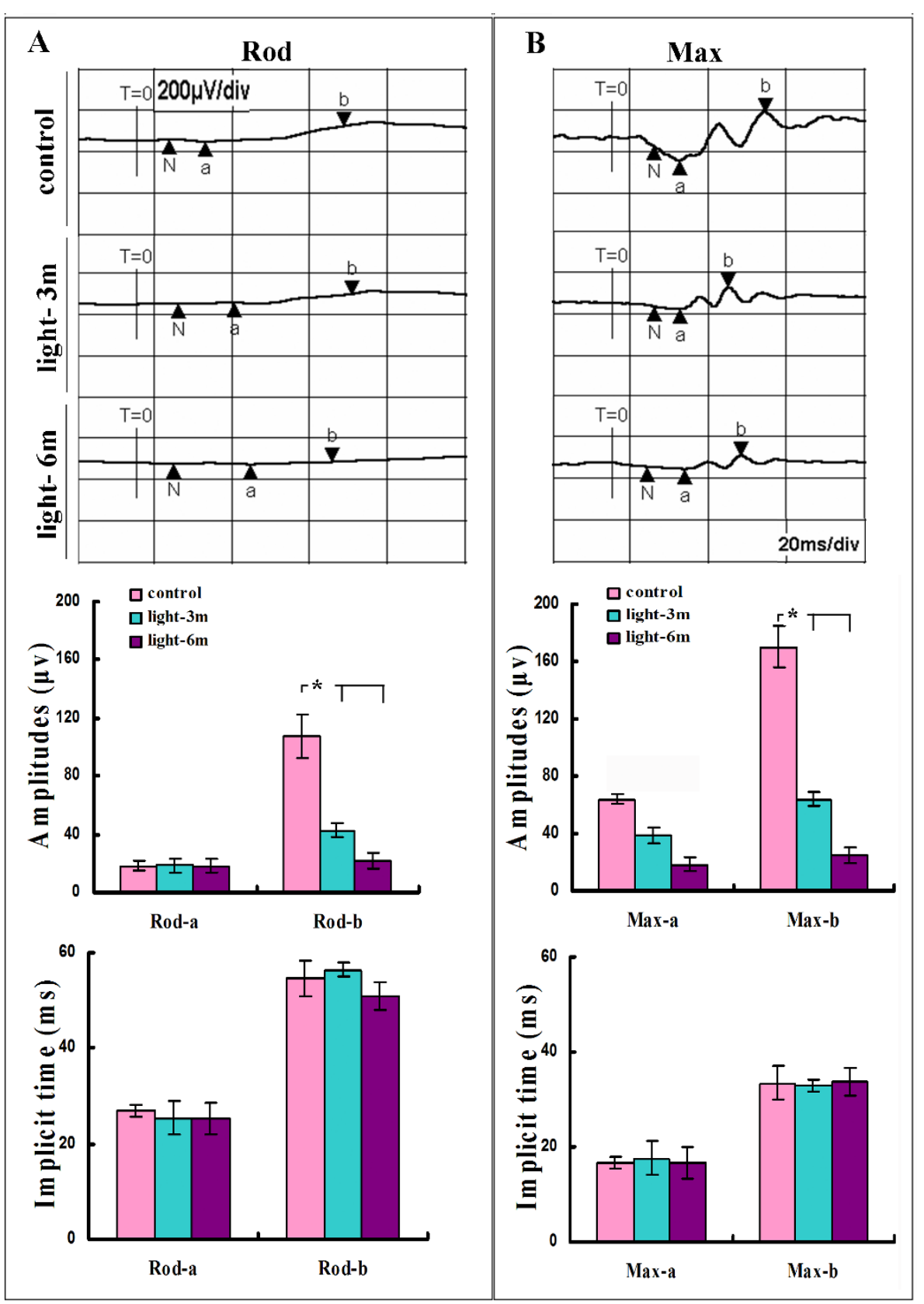

Figure 5 Electroretinogram analysis in APPswe/PS1 bigenic mice. Dark-adapted ERG amplitudes were measured in control and groups received 3-month (light-3 $\mathrm{m}$ ) and 6-month (light-6 m) CILEs as described in the Methods. (A) The rod-isolated ERG response stimulated by a flash at $0.008 \mathrm{~cd} / \mathrm{m} 2 \mathrm{~s}(-25 \mathrm{~dB})$. (B) The maximal ERG response by a flash at $2.5 \mathrm{~cd} / \mathrm{m}^{2} \mathrm{~s}(0 \mathrm{~dB})$. T indicates start of the test; $\mathrm{N}$, the baseline of the recording when light stimulation was given. Quantifications include measures from 12 mice for each group, i.e., $N=12$. *: $P<0.001$, Bars depict mean \pm SEM

uncover the molecular basis of light-induced $\mathrm{A} \beta$ accumulation and related photoreceptor degeneration.

\section{Conclusion}

In summary, our observations demonstrate degenerative changes in the outer retina with accumulation of $A \beta$ deposits, CNV and dramatically exaggerated neuroinflammatory response in the APPswe/PS1 bigenic mouse challenged by CILE. These results suggest that an Alzheimer's transgenic animal model with accumulation of $A \beta$ deposits might be an alternative animal model for AMD, if combined with other confounding factors for AMD. 


\section{Methods}

\section{Antibodies and other reagents}

Antibodies used in this study are listed in Table 1 in the Additional file 1 . Tropicamide and $0.5 \%$ proparacaine were obtained from Xingqi Pharmacectical (Shengyang, China) and Alcon Laboratories (Fort Worth, Texas), respectively. The primary antibody dilution buffer, the avidin-biotin complex $(\mathrm{ABC})$ kit for 3-Amino-9-ethylcarbazole (AEC) staining was bought from Boster (Wuhan, China), and 4,6-diamidino-2-phenylindole (DAPI) was purchased from Vector Laboratories (Burlingame, CA). Rodamine-conjugated phalloidin was obtained from Invitrogen (Carlsbad, CA). Total protein assay kit was from Bio-Rad (Hercules, CA). The enhanced chemiluminescence (ECL) detection system and the horse radish peroxidase-conjugated secondary antibody were purchased from Cell Signaling (Danvers, MA). Hematoxylin and eosin $(H \& E)$, and all other chemical reagents used in the experiments, unless indicated, were purchased from SigmaAldrich (St. Louis, MO).

\section{Animal and treatment}

A breeding pair of the bigenic mouse line that harbors a human amyloid precursor protein with Swedish mutations (K595N/M596L, APPswe) and a mutant human presenilin 1 (PS1-dE9, PS1) were obtained from the Jackson Laboratory (Bar Harbor, ME, USA) [44]. As described, this mouse line was originally derived from a hybrid of C57BL/ $6 \mathrm{~J}$ and $\mathrm{C} 3 \mathrm{H} / \mathrm{HeJ}$, which carries rd1 mutation (Pde6brd1) that confounds spontaneous retinal degeneration. To exclude possible disturbance of rd1 gene, all $r d 1$-positive mice were identified by PCR genotyping and excluded in breeding (see Additional file 1). Mice were normally maintained in the institutional transgenic mouse facility with 12/12 h light-dark cycle with food and water ad libitum. APPswe/PS1 bigenic mice and none-transgenic littermates (non-Tg) at the age of 6 months were grouped $(N=12)$ for treatments. When the groups of control mice were kept in normal conditions, CILE for the treated groups of mice was replaced by a source of 10,000-Lux cool full spectrum light (wavelength ranges from $380 \mathrm{~nm}-780$ $\mathrm{nm}$ ) for 3 months (light-3 m) or 6 months (light-6 m). The light intensity exposed to the animals was confirmed by a light meter (Thermo Fisher Scientific, Pittsburgh, PA). Temperature in animal cages during the light exposure was maintained between $22-24^{\circ} \mathrm{C}$. To ensure the efficacy of light exposure, both eyes of each mouse were also topically given $1 \%$ atropine long-lasting emulsion once a week during the entire period of CILE. All animal procedures were conducted in accordance with the guidelines of the Association for Research in Vision and Ophthalmology Statement for the Use of Animals in Ophthalmic and Vision Research and an approval of the Institutional
Animal Care and Use Committee (IACUC) of the Zhongshan Ophthalmic Center of Sun Yet-sen University.

\section{Fundus photography}

Fundus photography was performed as described with minor modification [19]. Briefly, following papillary dilation with $1 \%$ tropicamide and $2.5 \%$ phenylephrine in $\mathrm{HCl}$ solution and sedation with $4.3 \%$ chloral hydrate in PBS (intraperitoneal injection, i.p.), mice were placed under the microscope operation system platform (OMS-800, Topcon, Japan) and fundus images were directly captured using a Canon G9 digital camera attached to the scope over the eye that was covered by one drop of $1 \%$ hydroxypropyl methylcellulose over the cornea to compromise refractor errors.

\section{Electroretinogram analysis}

Scotopic electroretinogram (ERG) was recorded as reported previously [34]. Each group of animals was assayed with a RETIPORT ERG recording system (RETI Technologies, Gaithersburg, Germany). Before measuring, all animals were kept in dark overnight for at least 8 hours for dark-adaptation. Following papillary dilation with $1 \%$ tropicamide and $2.5 \%$ phenylephrine in $\mathrm{HCl}$ solution, sedation with $4.3 \%$ chloral hydrate in PBS (i.p.) and corneal anesthesia with $0.5 \%$ proparacaine, the gold loop electrodes were placed on the cornea, the reference electrode was plug into the mouth underneath of the tongue, and a ground electrode was subcutaneously inserted into the midway of the tail. The rod-only responses were recorded after stimulation by white light flashes with intensity of $0.008 \mathrm{~cd} / \mathrm{m} 2 \mathrm{~s}(-25 \mathrm{~dB})$, whereas the maximal response from the mixed rod/cone- responses (max response) was recorded with light flashes of $2.5 \mathrm{~cd} / \mathrm{m} 2 \mathrm{~s}(0 \mathrm{~dB})$. Three independent stimuli within $3 \mathrm{sec}$ intervals were recorded as a single ERG recording. At least 3 recordings were obtained from each eye. The amplitudes and the latency of $a$ and $b$ waves were measured.

\section{Tissue preparation}

Mice were euthanized with $4.3 \%$ chloral hydrate $(0.1 \mathrm{ml} / \mathrm{g})$ followed by transcardiac perfusion with ice-cold $0.1 \mathrm{M}$ phosphate buffered saline (PBS, pH 7.4). Eyes were then immediately enucleated and fixed in $4 \%$ paraformaldehyde (PFA) in PBS (pH7.4) overnight at room temperature (RT) or dissected to extract retina for preparation of tissue lysates for Western blots. Fixed eyes were then preserved in PBS containing 20\% sucrose and $0.05 \%$ sodium azide at $4^{\circ} \mathrm{C}$, gradually dehydrated in $70 \%-100 \%$ isopropanol, and embedded in paraffin blocks. Retinal cross sections $(4 \mu \mathrm{m}$ thickness) through the center of pupil-optic nerve head were prepared for histopathological analysis. To be prepared for transmission electron microscopy (EM), about 2 
$\mathrm{mm} \times 2 \mathrm{~mm}$ size of retina with attached RPE/choroid/ sclera was dissected from the proximity of the optic nerve head (within $5 \mathrm{~mm}$ range) from some enucleated eye cups following PBS perfusion. Dissected retina-chorid-sclera tissues were immediately fixed in $2.5 \%$ glutaraldehyde and $1 \%$ PFA in $0.1 \mathrm{M}$ sodium cacodylate- $\mathrm{HCl}(\mathrm{pH} 7.4)$ ( $24 \mathrm{~h}$ at $4^{\circ} \mathrm{C}$ ) followed by further post-fixation in $1 \%$ osmium tetroxide in $0.1 \mathrm{M}$ cacodylate buffer- $\mathrm{HCl}(\mathrm{pH} \mathrm{7.4)}$ for $4 \mathrm{~h}$, gradually dehydrated in 50\%-100\% alcohol followed by immersion in propylene oxide, and embedded in epoxy resin for preparation of ultrathin sections. RPE/choroid flatmounts were prepared as described previously [45]. In general, fixed eyes were split equatorially and retinas were carefully removed from the eyecup under a stereoscope. After the extraocular muscles were removed, the posterior eye segment containing the RPE/choroid complex of each eye was spread into four quarters by four radial cuts.

\section{Histopathology, immunohistochemistry and immunofluorescence microscopy}

Hematoxylin and eosin (H\&E) staining was performed for general histopathological assessment as previously described [8]. For immunohistochemistry, paraffinembedded retinal cross sections were deparaffinized, rehydrated, and autoclaved $\left(121^{\circ} \mathrm{C} \times 5\right.$ minutes in $10 \mathrm{mM}$ citrate buffer, $\mathrm{pH}$ 6.0) for antigen retrieval. Following quenching of endogenous peroxidase activity with 3\% $\mathrm{H}_{2} \mathrm{O}_{2}$ (20 min at $\mathrm{RT}$ ), sections were blocked with $5 \%$ goat serum in PBS containing 0.1\% Triton-X 100 and $20 \mathrm{mM}$ L-lysine incubated with an appropriate primary antibody as listed in Table 1 (Additional file 1 ) at $37^{\circ} \mathrm{C}$ for $1 \mathrm{~h}$. Specific immunoreactivity was then visualized by microscopy following incubation with a biotinylated secondary antibody, $A B C$ kit and $A E C$ staining. To detect $A ß$, outer retina flatmounts were treated by $70 \%$ formic acid for 5 min, washed with PBS, blocked with $4 \%$ bovine serum albumin in PBS containing 0.1\% Triton X-100 (30 min, $\mathrm{RT}$ ), and incubated with an appropriate primary antibody in primary antibody dilution buffer overnight at $4^{\circ} \mathrm{C}$. Following appropriate washing with PBS, retinal flatmounts were incubated with an appropriate fluorophore-conjugated second antibody, counterstained with DAPI, coverslipped onto microslides, and visualized using a Zeiss LSM510 Meta Confocal Microscope. For quantification, four non-overlapped areas were randomly selected along the equatorial zone which is about $300 \mu \mathrm{m}$ away from the optic disc, under the magnification of 400X. Images were captured using an Axioplan 2, Zeiss camera and further quantified with Image Pro-Plus1.42q (National Institutes of Health, USA). Total Aß-positive area, number of IBA1-immunoractive cells, and total length of IBA-1-immunoractive dendrites of microglia within the four selected regions were quantified. Disorganization of RPE alignment was scored as percentage of phalloidin-negative area over the entire RPE flat mounts.

\section{Western blot}

Western blotting was conducted as previously described [46,47]. Briefly, dissected retinas were homogenized in lysis buffer (50 mM Tris- $\mathrm{HCl} / \mathrm{pH} 7.5,5 \mathrm{mM}$ EDTA, 150 $\mathrm{mM} \mathrm{NaCl}, 0.5 \% \mathrm{NP}-40$, protease inhibitor cocktail mixture) and pelleted. The concentrations of total protein in the supernatants were assayed using Bio-Rad Protein Assay kit. About $35 \mu \mathrm{g}$ total protein of lysate from each samples was resolved in 10\% SDS-polyacrylamide gel, blotted on PVDF membrane followed by incubation with Blotto (0.1\% Tween-20 and 5\% milk in PBS, $\times 1 \mathrm{~h})$, a specific primary antibody in 5\% milk in PBST (overnight at $4^{\circ} \mathrm{C}$ ) and with a horse radish peroxidase-conjugated secondary antibody in RT with appropriate washing. The immunoreactivity was visualized with ECL.

\section{Transmission electron microscopy}

The ultrathin $(70 \mathrm{~nm})$ sections were prepared using a Leica UltraCut S Microtome, counterstained with uranyl acetate and lead citrate, and examined under a JEOL 100CX II electron microscope (JEOL, Tokyo, Japan) at $80 \mathrm{kV}$. Microscopic images were acquired using X-ray films.

\section{Statistical analysis}

In all of the graphs, the data points represent the means \pm S.E.M. from all individuals in each group of animals $(N=6-12)$. Applicable comparisons were performed by one-way analysis of variance followed by Student's $t$-test for multiple groups or independent samples or $T$-test for two groups by SPSS13.0 software. The difference between groups was considered as statistically significant when the value of $\mathrm{p}$ was $\leq 0.05$.

\section{Additional material}

Additional file 1: Supplementary data [48-54].

\section{Abbreviations}

APP: Amyloid precursor protein; AB: Beta-amyloid; AMD: Age-related macular degeneration; APPswe: Human amyloid precursor protein with Swedish mutations; BrM: Bruch's membrane; AEC: 3-Amino-9-ethylcarbazole; CILE: Cyclic intensive light exposure; CNV: Choroidal neovascularization; CC: Choroicapillaris; DAPI: 4,6-diamidino-2-phenylindole; ERG: Electroretinogram; ECL: Enhanced chemiluminescence; EM: Transmission electron microscopy; H\&E: Hematoxylin and eosin; IBA-1: Ionized calcium binding adaptor molecule-1; IACUC: The Institutional Animal Care and Use Committee; INL: Inner nuclear layer; IPL: Inner plexiform layer; non-Tg: None transgenic littermates; OPL: Outer plexiform layer; ONL: Out nuclear layer; OS: Photoreceptor outer segment; OCL: Outer collagenous layer; PS1: Presenilin 1; PCR: Polymerase chain reaction; PFA: Paraformaldehyde; PBS: Phosphate 
buffered saline; RT: Room temperature; RGC: Retinal ganglion cell; RPE: Retinal pigment epithelium; VEGF: Vascular endothelial growth factor.

\section{Acknowledgements}

The study was supported by National Basic Research Program of China (973) 2007CB512207 and National Natural Science Foundation of China (30973266) to JG and University of California Irvine Alzheimer's Disease Research Center Grant to ZT.

\section{Author details}

${ }^{1}$ State Key Laboratory of Ophthalmology, Zhongshan Ophthalmic Center, Sun Yat-sen University, Guangzhou, China. 'Department of Neurology, University of California Irvine School of Medicine, Irvine, CA 92697, USA. ${ }^{3}$ Institute for Memory Impairments and Neurological Disorders, University of California Irvine School of Medicine, Irvine, CA 92697, USA.

\section{Authors' contributions}

$\mathrm{ZD}$ and $J \mathrm{~L}$ conceived of the study and experimental design and drafted manuscript. ZD and $J \mathrm{~L}$ carried out experiments. $Z D, J L, Y L, X L, H H$ and $Y H$ helped experimental design and data analysis. ZT initiated original idea for the study and steered data presentation and manuscript drafting and revision. JG funded the study, guided conceiving of the study and experiments, and helped data analysis, manuscript drafting and revision. All authors read and approved the final manuscript.

\section{Competing interests}

The authors declare that they have no competing interests.

Received: 9 December 2011 Accepted: 24 March 2012

Published: 24 March 2012

\section{References}

1. de Jong PT: Age-related macular degeneration. N Engl J Med 2006, 355(14):1474-1485

2. Hageman GS, Gehrs K, Johnson LV, Anderson D: Age-Related Macula Degeneration (AMD).Edited by: Kolb H, Fernandez E, Nelson R. Webvision: The Oragnization of the Retina and Visual System; 2008:[http://www.ncbi. nlm.nih.gov/books/NBK27323/], Salt Lake City (UT): University of Utah Health Sciences Center, 1995.

3. Kaarniranta K, Salminen A, Haapasalo A, Soininen H, Hiltunen M: AgeRelated Macular Degeneration (AMD): Alzheimer's Disease in the Eye? Alzheimers Dis 2011, 24(4):615-631.

4. Luibl V, Isas JM, Kayed R, Glabe CG, Langen R, Chen J: Drusen deposits associated with aging and age-related macular degeneration contain nonfibrillar amyloid oligomers. J Clin Invest 2006, 116(2):378-385.

5. Dentchev T, Milam AH, Lee VM, Trojanowski JQ, Dunaief JL: Amyloid-beta is found in drusen from some age-related macular degeneration retinas, but not in drusen from normal retinas. Mol Vis 2003, 9:184-190.

6. Isas JM, Luibl V, Johnson LV, Kayed R, Wetzel R, Glabe CG, Langen R, Chen J: Soluble and mature amyloid fibrils in drusen deposits. Invest Ophthalmol Vis Sci 2010, 51(3):1304-1310.

7. Johnson LV, Leitner WP, Rivest AJ, Staples MK, Radeke MJ, Anderson DH: The Alzheimer's A beta -peptide is deposited at sites of complement activation in pathologic deposits associated with aging and age-related macular degeneration. Proc Natl Acad Sci USA 2002, 99(18):11830-11835.

8. Liu B, Rasool S, Yang Z, Glabe CG, Schreiber SS, Ge J, Tan Z: Amyloidpeptide vaccinations reduce $\{$ beta\}-amyloid plaques but exacerbate vascular deposition and inflammation in the retina of Alzheimer's transgenic mice. Am J Pathol 2009, 175(5):2099-2110.

9. Malek G, Johnson LV, Mace BE, Saloupis P, Schmechel DE, Rickman DW Toth CA, Sullivan PM, Bowes Rickman C: Apolipoprotein E alleledependent pathogenesis: a model for age-related retinal degeneration. Proc Natl Acad Sci USA 2005, 102(33):11900-11905.

10. Ding JD, Lin J, Mace BE, Herrmann R, Sullivan P, Bowes Rickman C: Targeting age-related macular degeneration with Alzheimer's disease based immunotherapies: anti-amyloid-beta antibody attenuates pathologies in an age-related macular degeneration mouse model. Vision Res 2008, 48(3):339-345.

11. Ding JD, Johnson LV, Herrmann R, Farsiu S, Smith SG, Groelle M, Mace BE, Sullivan P, Jamison JA, Kelly U, Harrabi O, Bollini SS, Dilley J, Kobayashi D, Kuang B, Li W, Pons J, Lin JC, Rickman CB: Anti-amyloid therapy protects against retinal pigmented epithelium damage and vision loss in a model of age-related macular degeneration. Proc Natl Acad Sci USA 2011, 108(28):E279-E287.

12. Thornton J, Edwards R, Mitchell P, Harrison RA, Buchan I, Kelly SP: Smoking and age-related macular degeneration: a review of association. Eye (Lond) 2005, 19(9):935-944.

13. Tomany SC, Cruickshanks K, Klein R, Klein BE, Knudtson MD: Sunlight and the 10-year incidence of age-related maculopathy: the Beaver Dam Eye Study. Arch Ophthalmol 2004, 122(5):750-757.

14. Klein R, Peto T, Bird A, Vannewkirk MR: The epidemiology of age-related macular degeneration. Am J Ophthalmol 2004, 137(3):486-495.

15. Organisciak DT, Vaughan DK: Retinal light damage: mechanisms and protection. Prog Retin Eye Res 2010, 29(2):113-134

16. Wenzel A, Grimm C, Samardzija M, Reme CE: Molecular mechanisms of light-induced photoreceptor apoptosis and neuroprotection for retinal degeneration. Prog Retin Eye Res 2005, 24(2):275-306.

17. Duncan $J \mathrm{~L}$, LaVail MM: Intense cyclic light-induced retinal degeneration in rats. Arch Ophthalmol 2010, 128(2):244-245.

18. Albert DM, Neekhra A, Wang S, Darjatmoko SR, Sorenson CM, Dubielzig RR, Sheibani N: Development of choroidal neovascularization in rats with advanced intense cyclic light-induced retinal degeneration. Arch Ophthalmol 2010, 128(2):212-222.

19. Imamura Y, Noda S, Hashizume K, Shinoda K, Yamaguchi M, Uchiyama S, Shimizu T, Mizushima Y, Shirasawa T, Tsubota K: Drusen, choroidal neovascularization, and retinal pigment epithelium dysfunction in SOD1deficient mice: a model of age-related macular degeneration. Proc Nat Acad Sci USA 2006, 103(30):11282-11287.

20. Cousins SW, Espinosa-Heidmann DG, Alexandridou A, Sall J, Dubovy S, Csaky K: The role of aging, high fat diet and blue light exposure in an experimental mouse model for basal laminar deposit formation. Exp Eye Res 2002, 75(5):543-553.

21. Hollborn M, Francke M, landiev I, Buhner E, Foja C, Kohen L, Reichenbach A Wiedemann P, Bringmann A, Uhlmann S: Early activation of inflammationand immune response-related genes after experimental detachment of the porcine retina. Invest Ophthalmol Vis Sci 2008, 49(3):1262-1273.

22. Weber BH, Schrewe H, Molday LL, Gehrig A, White KL, Seeliger MW, Jaissle GB, Friedburg C, Tamm E, Molday RS: Inactivation of the murine Xlinked juvenile retinoschisis gene, Rs $1 \mathrm{~h}$, suggests a role of retinoschisin in retinal cell layer organization and synaptic structure. Proc Natl Acad Sci USA 2002, 99(9):6222-6227

23. Ning A, Cui J, To E, Ashe KH, Matsubara J: Amyloid-beta deposits lead to retinal degeneration in a mouse model of Alzheimer disease. Invest Ophthalmol Vis Sci 2008, 49(11):5136-5143.

24. Cai Z, Zhao B, Ratka A: Oxidative stress and beta-Amyloid protein in Alzheimer's disease. Neuromolecular Med 2011, 13(4):223-250.

25. Bressler SB: Introduction: understanding the role of angiogenesis and antiangiogenic agents in age-related macular degeneration. Ophthalmology 2009, 116(10 Suppl):S1-S7.

26. Bird AC: Therapeutic targets in age-related macular disease. J Clin Invest 2010, 120(9):3033-3041.

27. Cruickshanks KJ, Klein R, Klein BE, Nondahl DM: Sunlight and the 5-year incidence of early age-related maculopathy: the beaver dam eye study. Arch Ophthalmol 2001, 119(2):246-250.

28. LaVail MM, Gorrin GM, Repaci MA, Thomas LA, Ginsberg HM: Genetic regulation of light damage to photoreceptors. Invest Ophthalmol Vis Sci 1987, 28(7):1043-1048

29. Collier RJ, Wang Y, Smith SS, Martin E, Ornberg R, Rhoades K, Romano C: Complement deposition and microglial activation in the outer retina in light-induced retinopathy: inhibition by a 5-HT1A agonist. Invest Ophthalmol Vis Sci 2011, 52(11):8108-8116.

30. Marco-Gomariz MA, Hurtado-Montalban N, Vidal-Sanz M, Lund RD, VillegasPerez MP: Phototoxic-induced photoreceptor degeneration causes retinal ganglion cell degeneration in pigmented rats. J Comp Neurol 2006 498(2):163-179.

31. Palop JJ, Mucke L: Amyloid-beta-induced neuronal dysfunction in Alzheimer's disease: from synapses toward neural networks. Nat Neurosci 2010, 13(7):812-818.

32. Castellani RJ, Smith MA: Compounding artefacts with uncertainty, and an amyloid cascade hypothesis that is 'too big to fail'. J Pathol 2011, 224(2):147-152. 
33. Yoshida T, Ohno-Matsui K, Ichinose $S$, Sato T, Iwata N, Saido TC, Hisatomi T, Mochizuki M, Morita I: The potential role of amyloid beta in the pathogenesis of age-related macular degeneration. J Clin Invest 2005, 115(10):2793-2800.

34. Perez SE, Lumayag S, Kovacs B, Mufson EJ, Xu S: Beta-amyloid deposition and functional impairment in the retina of the APPswe/PS1DeltaE9 transgenic mouse model of Alzheimer's disease. Invest Ophthalmol Vis Sci 2009, 50(2):793-800.

35. Boscolo E, Folin M, Nico B, Grandi C, Mangieri D, Longo V, Scienza R, Zampieri P, Conconi MT, Parnigotto PP, Ribatti D: Beta amyloid angiogenic activity in vitro and in vivo. Int J Mol Med 2007, 19(4):581-587.

36. Biron KE, Dickstein DL, Gopaul R, Jefferies WA: Amyloid triggers extensive cerebral angiogenesis causing blood brain barrier permeability and hypervascularity in Alzheimer's disease. PLoS One 2011, 6(8):e23789.

37. Ni YQ, Xu GZ, Hu WZ, Shi L, Qin YW, Da CD: Neuroprotective effects of naloxone against light-induced photoreceptor degeneration through inhibiting retinal microglial activation. Invest Ophthalmol Vis Sci 2008, 49(6):2589-2598

38. Santos AM, Martin-Oliva D, Ferrer-Martin RM, Tassi M, Calvente R, Sierra A, Carrasco MC, Marin-Teva JL, Navascues J, Cuadros MA: Microglial response to light-induced photoreceptor degeneration in the mouse retina. J Comp Neurol 2010, 518(4):477-492.

39. Zhang C, Lei B, Lam TT, Yang F, Sinha D, Tso MO: Neuroprotection of photoreceptors by minocycline in light-induced retinal degeneration. Invest Ophthalmol Vis Sci 2004, 45(8):2753-2759.

40. El Khoury J, Luster AD: Mechanisms of microglia accumulation in Alzheimer's disease: therapeutic implications. Trends Pharmacol Sci 2008, 29(12):626-632.

41. Fiala M, Zhang L, Gan X, Sherry B, Taub D, Graves MC, Hama S, Way D, Weinand M, Witte M, Lorton D, Kuo YM, Roher AE: Amyloid-beta induces chemokine secretion and monocyte migration across a human bloodbrain barrier model. Mol Med 1998, 4(7):480-489.

42. Davis JB, McMurray HF, Schubert D: The amyloid beta-protein of Alzheimer's disease is chemotactic for mononuclear phagocytes. Biochem Biophys Res Commun 1992, 189(2):1096-1100.

43. Harada T, Harada C, Kohsaka S, Wada E, Yoshida K, Ohno S, Mamada H, Tanaka K, Parada LF, Wada K: Microglia-Muller glia cell interactions control neurotrophic factor production during light-induced retinal degeneration. J Neurosci 2002, 22(21):9228-9236.

44. Jankowsky $J$, Slunt HH, Ratovitski T, Jenkins NA, Copeland NG, Borchelt DR: Co-expression of multiple transgenes in mouse CNS: a comparison of strategies. Biomol Eng 2001, 17(6):157-165.

45. Bruban J, Glotin AL, Dinet V, Chalour N, Sennlaub F, Jonet L, An N, Faussat AM, Mascarelli F: Amyloid-beta(1-42) alters structure and function of retinal pigmented epithelial cells. Aging Cell 2009, 8(2):162-177.

46. Li J, Dong Z, Liu B, Zhuo Y, Sun X, Yang Z, Ge J, Tan Z: Hypoxia induces beta-amyloid in association with death of RGC-5 cells in culture. Biochem Biophys Res Commun 2011, 410(1):40-44.

47. Hoh Kam J, Lenassi E, Jeffery G: Viewing ageing eyes: diverse sites of amyloid Beta accumulation in the ageing mouse retina and the upregulation of macrophages. PLoS One 2010, 5(10), pii: e13127.

48. Martin LJ, Sisodia SS, Koo EH, Cork LC, Dellovade TL, Weidemann A, Beyreuther K, Masters C, Price DL: Amyloid precursor protein in aged nonhuman primates. Proc Natl Acad Sci USA 1991, 88(4):1461-1465.

49. Thakker DR, Weatherspoon MR, Harrison J, Keene TE, Lane DS, Kaemmerer WF, Stewart GR, Shafer LL: Intracerebroventricular amyloidbeta antibodies reduce cerebral amyloid angiopathy and associated micro-hemorrhages in aged Tg2576 mice. Proc Natl Acad Sci USA 2009, 106(11):4501-4506

50. Ito D, Imai Y, Ohsawa K, Nakajima K, Fukuuchi Y, Kohsaka S: Microgliaspecific localisation of a novel calcium binding protein, Iba1. Brain Res Mol Brain Res 1998, 57(1):1-9.

51. Wakisaka Y, Chu Y, Miller JD, Rosenberg GA, Heistad DD: Spontaneous intracerebral hemorrhage during acute and chronic hypertension in mice. J Cereb Blood Flow Metab 2010, 30(1):56-69.

52. Zhang B, Veasey SC, Wood MA, Leng LZ, Kaminski C, Leight S, Abel T, Lee VM, Trojanowski JQ: Impaired rapid eye movement sleep in the Tg2576 APP murine model of Alzheimer's disease with injury to pedunculopontine cholinergic neurons. Am J Pathol 2005, 167(5):1361-1369.
53. Pratico D, Uryu K, Leight S, Trojanoswki JQ, Lee VM: Increased lipid peroxidation precedes amyloid plaque formation in an animal model of Alzheimer amyloidosis. J Neurosci 2001, 21(12):4183-4187.

54. Bowes C, Li T, Frankel WN, Danciger M, Coffin JM, Applebury ML, Farber DB: Localization of a retroviral element within the rd gene coding for the beta subunit of cGMP phosphodiesterase. Proc Natl Acad Sci USA 1993, 90(7):2955-2959.

doi:10.1186/1471-2202-13-34

Cite this article as: Dong et al:: Cyclic intensive light exposure induces retinal lesions similar to age-related macular degeneration in APPswe/ PS1 bigenic mice. BMC Neuroscience 2012 13:34.

\section{Submit your next manuscript to BioMed Central and take full advantage of:}

- Convenient online submission

- Thorough peer review

- No space constraints or color figure charges

- Immediate publication on acceptance

- Inclusion in PubMed, CAS, Scopus and Google Scholar

- Research which is freely available for redistribution

Submit your manuscript at www.biomedcentral.com/submit
C Biomed Central 